\title{
Production, obtention et valorisation des beurres exotiques
}

Xavier PAGES

Responsable Conseil et Transfert, Responsable projet Technologie et Lipochimie, ITERG, 11 rue Gaspard Monge, Bersol 2, 33600 Pessac $<x$.pages@iterg.com>

$<w w w . i t e r g . c o m>$
"La vérité est comme une braise ardente, elle brûle qui ne l'a pas comprise. Pour être saisie, elle doit être enveloppée dans quelque chose "

Sur les traces d'Amkoullel l'enfant Peul Amadou Hampate Ba

Par définition, les beurres végétaux sont des corps gras solides à température ambiante.

De nombreux beurres d'origine végétale sont disponibles en Afrique et dans les pays tropicaux et présentent des propriétés intéressantes du point vue de leur valorisation.

\begin{abstract}
By definition, "butters" are anhydrous and solid fats at room temperature. A lot of different crude fats and butters are available in Africa and other tropical countries, and their characteristics make often them interesting to valorise. This presentation will focus on a personal experience dealing with production and development of African butters. The production of butters and particularly the shea fat, is done by one of the two basic methods, roughly described as "traditional or modern". The rural (traditional) vegetable oil extraction processes, which predominate the extraction technologies in many of the shea kernel producing areas, involve water extraction. This method often employs simple small sized and household equipments; it is based on aqueous extraction techniques to produce oil. The work is very hard but provides jobs, money and "consideration" to the women who are traditionally in charge of this work. Such a traditional method yields not more than $35 \%$ of the kernel oil contents and the product quality is often poor. It is obvious that a major contributing factor to the low yield is the treatment inability to adequately break the kernel cellular structures, a necessity to obtain an efficient oil/fat extraction. The "modern" methods involve the use of mechanical presses and organic solvents; although the methods are quite high-yielding (with extraction rates around $80 \%$ ), they are reportedly difficult to perform because of the high latex content in the kernels: in fact the latex agglutinates and clogs the equipment or inhibits an efficient penetration of the extraction solvent. As we all know olein rich vegetable oils are globally abundant but commercial sources of vegetable fats, or "stearin", are less common. Stearin is used in confectionery for chocolates, cakes, etc. and for margarine industries. The most developed and well-known source of stearin is cocoa butter; the development of this production is historical and is based on the "chocolate business" involving a lot of organisms and industries mainly located in "developed" countries. Beside this, other butters like Shea butter, Illipe, Kokum, Sal and mangoare available sources for substitutes in the production of cocoa butter improvers $(C B I)$, equivalent (CBE) or substitutes (CBS). Other markets for Shea butter exist in the personal care industry; the main reason for this growing interest has been the recognition by the cosmetic industry and consumers of the Sheat butter therapeutic benefits (ultra violet light protection, antiinflammatory, moisturizing, regenerative, anti-eczema and anti-wrinkle properties) due to the presence of a significant fraction of unsaponifiable matters (3-15 \%) composed of many bioactive chemicals, e.g. triterpene alcohols, phenols, sterols and the poly-isoprenic hydrocarbons like karitenes. In this field of valorisation (cosmetics), the unsaponifiable content is a key factor. In 1992 on the demand of DESLOG company, ITERG worked and patented an interesting method of increasing the unsaponifiable content of Shea butter using cryogenic enrichment by fractionation at about $-30^{\circ} \mathrm{C}$. The final product called CRYOKARITENE (registered trade mark) can be enriched up to more than $50 \%$ of unsaponifiable and shows excellent dermo-cosmetic properties.
\end{abstract}

Key words: topical butter, shea, production, refining CBE

En Europe, mis à part les produits d'origine animale, nous ne disposons pas de matières premières grasses solides à la température ambiante. D'où le recours obligé à l'hydrogénation qui s'y pratique, et ceci en l'absence (et dans l'attente hypothétique) d'huiles innovantes qui seraient dotées de fractions solides (comme le seraient par exemple des huiles de tournesol stéarique). Pour l'heure donc et pour satisfaire aux faibles taux de « trans » souhaités par les marchés du Nord, ces graisses solides, ces « beurres » venus du Sud, sont de fait largement utilisés par I'industrie du chocolat, la margarinerie, la biscuiterie, la cosmétique, etc.
Nous traiterons ici des beurres africains, des problèmes auxquels ils sont confrontés et des solutions qui pourraient être mises au service de leur production, tels qu'ils ressortent d'une expérience personnelle de plusieurs années sur le terrain.

\section{Les beurres natifs}

Les beurres natifs, c'est-à-dire extraits de noyaux ou de graines et dont les constituants principaux sont des triglycérides, sont très nombreux. Une de ces sources les plus connues 
est le beurre de cacao dont la production a été et demeure entièrement liée aux besoins de l'industrie chocolatière européenne et nord-américaine ${ }^{1}$. D'autres bases comme le karité, l'illipé, le sal, sont parfois utilisées permettant d'obtenir d'excellents substituts. Un tableau des différentes matières grasses d'origine végétale présentant des chaînes grasses saturées a été dressé par Émile André en 1947 [2]. Ànoter l'intérêt de la famille des sapotacées (sapotaceous), famille dans laquelle tous les parenchymes contiennent des cellules lacticifères, famille appartenant exclusivement aux régions tropicales incluant aussi les gommes (guttas et guttoides, balata, chicle); des huiles et graisses, et un fruit estimé, la sapotille.

Outre le beurre de Karité (Butyrospermum Parkii, Kotschy du Soudan) et dans cette même famille des sapotacées, ont été ainsi caractérisés et cités par $\mathrm{E}$. André les beurres de :

- Dumouri, Dumouria Hecklii, A. Chev (Côte d'Ivoire) ;

- Makoré, Baillonella toxisperma, Pierre (Côte d'Ivoire) ;

- Noungou, Thiegemella africana, Pierre (Côte d'Ivoire);

- Djavé, Minusops Djavé, Engler (Cameroun);

- Moabi, Minusops Pierreana, Engler (Gabon) ;

- L'Illipé, Bassia longifolia, Willd (Indes);

- Mohwra, Bassia latifolia, Roxb. (Indes);

- Fulware, Bassia butyracea, Roxb. (Indo-Chine). Potentiellement, du fait de la présence de ces cellules lactifères, tous ces beurres peuvent présenter un intérêt en cosmétologie.

\section{Le beurre de karité}

Le beurre de karité a joué de tout temps et joue toujours un rôle important dans la vie des villages africains où il est traditionnellement utilisé comme source d'huile végétale en alimentation mais également en cosmétique, pour la fabrication de crème, de savon.

Sa découverte (par les Européens) est ancienne. Ainsi au XVIII ${ }^{\text {e }}$ siècle, à la recherche de l'or, un directeur de la Compagnie normande pour l'exploitation du commerce du Sénégal, André Brüe, rapporte qu'au cours d'une de ses pérégrinations commerciales, le long du fleuve Sénégal, au sein du royaume de Golam, «les marchands marabouts lui donnèrent plusieurs calebasses remplies d'une certaine graisse un peu moins blanche que le suif de mouton et à peu près de la même consistance; ils I'appellent Bataula dans le pays. Les nègres du bas de la rivière l'appellent BamboukToulou, c'est-à-dire beurre de Bambouk (province) (...) Les nègres se servent de cette

\footnotetext{
${ }^{1}$ Voir pour une analyse des vicissitudes de ce marché en régime de mondialisation libérale l'article de Bruno Losch [1].
}

graisse comme nous nous servons du beurre ou du saindoux en France (...). Mais on l'emploie plus ordinairement avec un succès merveilleux plus prompt et plus sûr que celui de I'huile de palme à la guérison des rhumatismes. II suffit d'en frotter les parties affligées afin de faire pénétrer la graisse ... Les chirurgiens français se sont avisés d'y mêler de l'esprit de vin ou de l'eau-de-vie, mais les nègres prétendent qu'il vaut mieux boire l'eau-de-vie que de l'employer à cet usage » [3].

Mais c'est Mungo Park-un voyageur naturaliste écossais qui périt victime de sa passion des voyages et d'une tribu de "nègres batailleurs "auquel la tradition se rapporte comme le véritable « inventeur» du beurre de karité. Dans son récit intitulé Voyage dans l'intérieur de I'Afrique, il note que : "...indépendamment des esclaves et des marchandises qu'ils portent pour les blancs, les marchands amènent sur la cote de Gambie, du fer natif, des gommes odorantes et du shétoulou, ce qui signifie littéralement beurre d'arbre ou beurre végétal. Ce beurre est extrait d'une espèce de noix, par le moyen de l'eau bouillante... il ressemble au beurre ordinaire et en a la consistance. Les habitants étaient toujours occupés à recueillir les fruits de l'arbre "shea "; cet arbre croit abondamment dans toute cette partie du bambara (...) il n'est pas planté par les habitants mais on le retrouve croissant naturellement dans les bois. Lorsque l'on défriche les forêts pour cultiver la terre, on coupe tous les arbres excepté les "sheas» [4]...

Un reste éloigné de cette tradition demeuret-il ? À Ouagadougou (Burkina Fasso), on fait maintenant une sculpture du bois de chaque arbre mort.

Sur le plan botanique, "l'arbre à beurre » (Butyrospermum Parkii) appartient à la famille des sapotacées.

L'arbre peut atteindre 12 à $20 \mathrm{~m}$ de hauteur et de 0,6 à 1,2 $\mathrm{m}$ de diamètre et rappelle le chêne pédonculé (quereuspedonculata). II entre en production après 20 ans, et connaît son plein rendement entre 40 et 50 ans (figure 1).
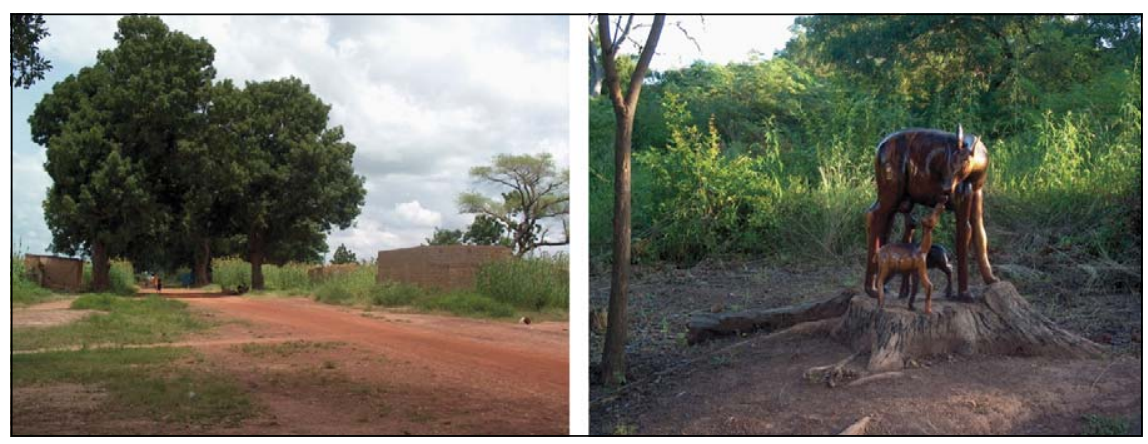

Figure 1. «Arbres à beurre », Burkina Fasso.
Plusieurs variétés ont été identifiées qui se distinguent en particulier par leurs teneurs en insaponifiable, les principales étant le :

- Mangifolia: variété à feuilles de manguier, présentant une teneur en insaponifiable de $5,1 \%$ à $16,6 \%$;

- Poissoni du Ghana (insaponifiable de 3,6 \% à $8,5 \%)$;

- Nilotica de l'Ouganda (insaponifiable 2,6 à $3 \%)$.

Enfin se rencontrent également cinq autres variétés: les variétés cuneata, serotina, parviflora se rattachant à la variété mangifolia et les variétés ferruginea, flocosa se rattachant à la variété Poissoni.

Les amandes (figure 2) contiennent jusqu'à $55 \%$ de matière grasse avec un taux élevé d'insaponifiable. À noter l'influence du degré de maturité de la graine, les graines immatures étant plus riches en insaponifiable.

\section{Utilisations}

Les utilisations du beurre de karité sont multiples.

II est utilisé en alimentation, localement mais aussi après exportation sur un plan industriel en biscuiterie, pâtisserie, chocolaterie, où pour ce dernier cas il peut intervenir comme substitut au beurre de cacao.

En cosmétique, de par sa teneur et composition en insaponifiable, les Égyptiens de l'Antiquité I'utilisait déjà (figure 3), pour la constitution de fards et rouges pour lèvre « il faut donner, naturellement la préférence aux corps gras qui ne rancissent pas. Parmi ceux-ci les plus agréables aux muqueuses sont évidemment le beurre de cacao ou de karité et les mélanges de ces beurres avec de I'huile de vaseline ».

En pharmacologie Berhaut (1975) signale par exemple I'utilisation des graines torréfiées et réduites en poudre de Jatropha Curcas, en mélange avec du piment (Capsicum frutescens) et du beurre de karité pour un cataplasme favorisant l'expulsion du ver de guinée. 


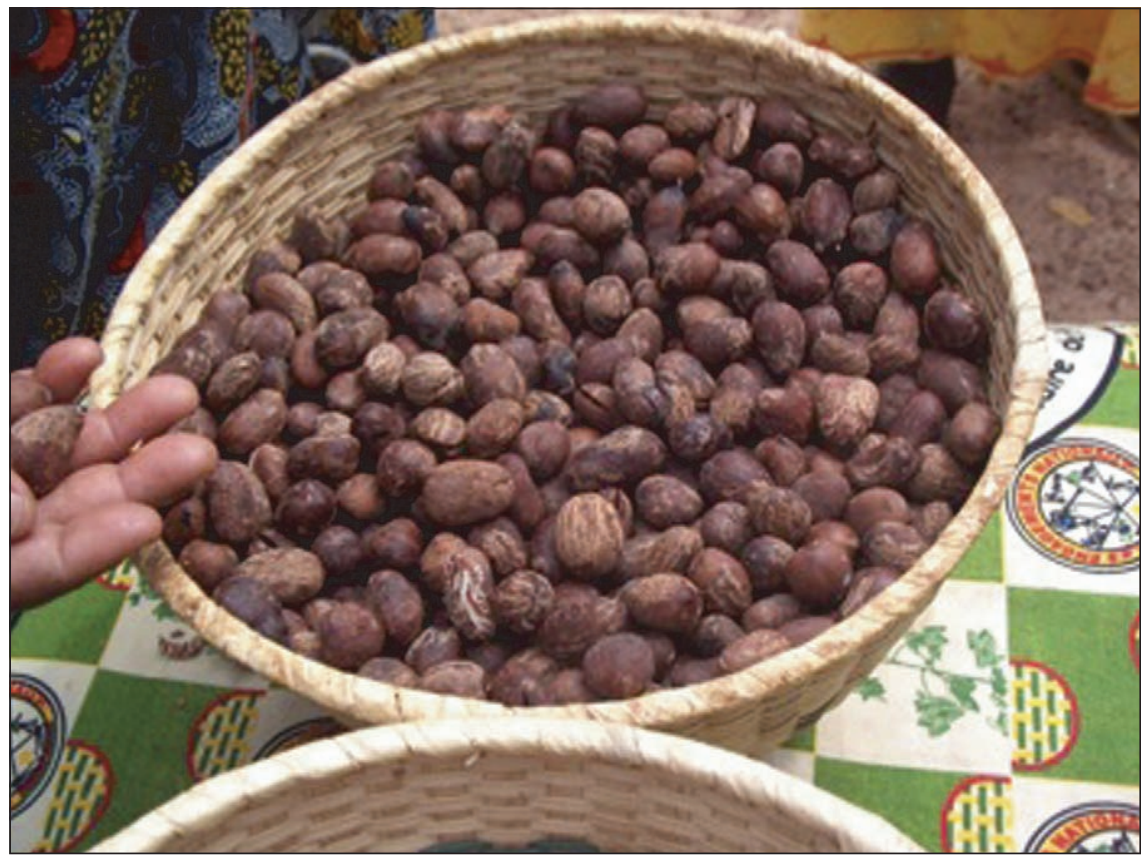

Figure 2. Les amandes contiennent 45 à $55 \%$ de MG.

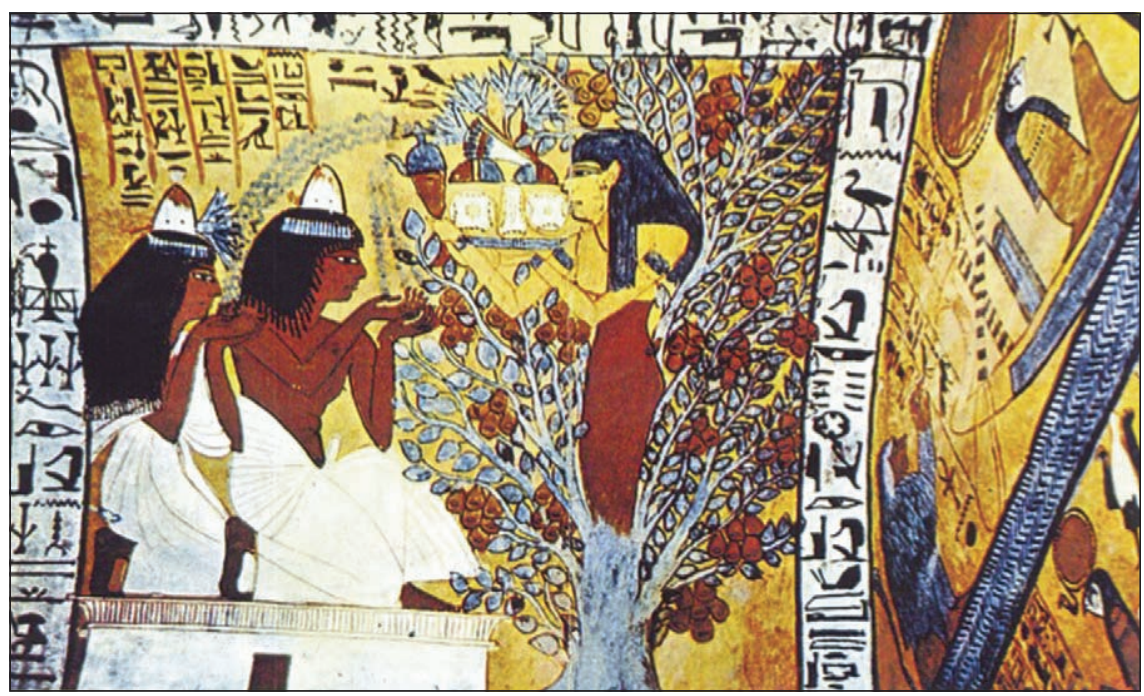

Figure 3. Tombeau de Senedjem, 1292 av. JC. La déesse Nout apparaît devant le couple défunt : sur leurs têtes une petite motte de baume à base de beurre de karité.

\section{Production : la méthode traditionnelle villageoise}

"L'intelligence est un fruit que l'on ramasse dans le jardin de son voisin "

Batawa : Zaïre

\section{Le travail des femmes}

Les fruits sont récoltés à maturité vers la mi-juin dans le haut Sénégal, en fait de mai à août selon les régions. Le traitement des fruits et la fabrica-

\section{Opérations préliminaires}

Une fois les fruits collectés intervient le dépulpage, suivi du décorticage c'est-à-dire la séparation des amandes des noix et leur conservation dans de bonnes conditions.

Dépulpage: les fruits sont placés le plus souvent dans des fosses (fermentation avec eau + argile puis séchés au soleil ou audessus du foyer des cases). On fait bouillir la noix dans une marmite en aluminium ce qui est une très bonne solution qui permet d'inactiver les enzymes qui pourraient hydrolyser les corps gras (inactivation des lipases). On peut aussi fumer la noix dans un four artisanal (la noix devient cassante), le latex est coagulé (germination et moisissures sont enrayées). Les graines sont ensuite retirées de la pulpe et mises à sécher au soleil.

Le décorticage (séparation des amandes des noix) s'effectue par pilage dans mortier ou pilon suivi d'un vannage pour séparer les amandes des coques. On concasse ensuite l'amande. Le concassage peut se faire de façon très traditionnelle avec pilon ou en utilisant des petits broyeurs.

\section{La préparation proprement dite}

Ces opérations effectuées, on peut procéder à la cuisson des amandes concassées. L'extraction du beurre (figure 5) s'obtient après incorporation de l'eau tiède à la pâte et pétrissage (hydratation).

Le barattage « lave » les agglomérats de beurre (eau chaude puis froide) puis le lissage améliore la décantation de l'eau (figure 6): le beurre blanc remonte à la surface.

Ces opérations pénibles sont effectuées à la main.

Suit une opération de purification : le beurre est porté à ébullition: une écume surnageante apparaît. Elle est fouettée puis éliminée : le beurre est récupéré. Les matières solides décantent (particules d'amandes, farine, impuretés...); les tourteaux peuvent être valorisés dans certaines sauces et aussi en construction (effet insecticide dans les cases en banco).

Le beurre est ensuite conditionné et stocké.

Cette méthode traditionnelle, qui connaît un certain nombre de variantes, présente des rendements assez variables, de l'ordre de $23 \%$ à $35 \%$ à partir d'amandes à $45 \%$ de matière grasse et $10 \%$ d'eau.

\section{Intérêt de certains équipements \\ "Celui qui vit près de l'eau sait nager »}

(Bashi : RDC)

10 ans et 50 femmes productrices, et produit entre 10 et 100 tonnes par an d'un excellent beurre avec l'appellation biologique.
Des équipements adéquats permettent de réduire considérablement les taches pénibles. 


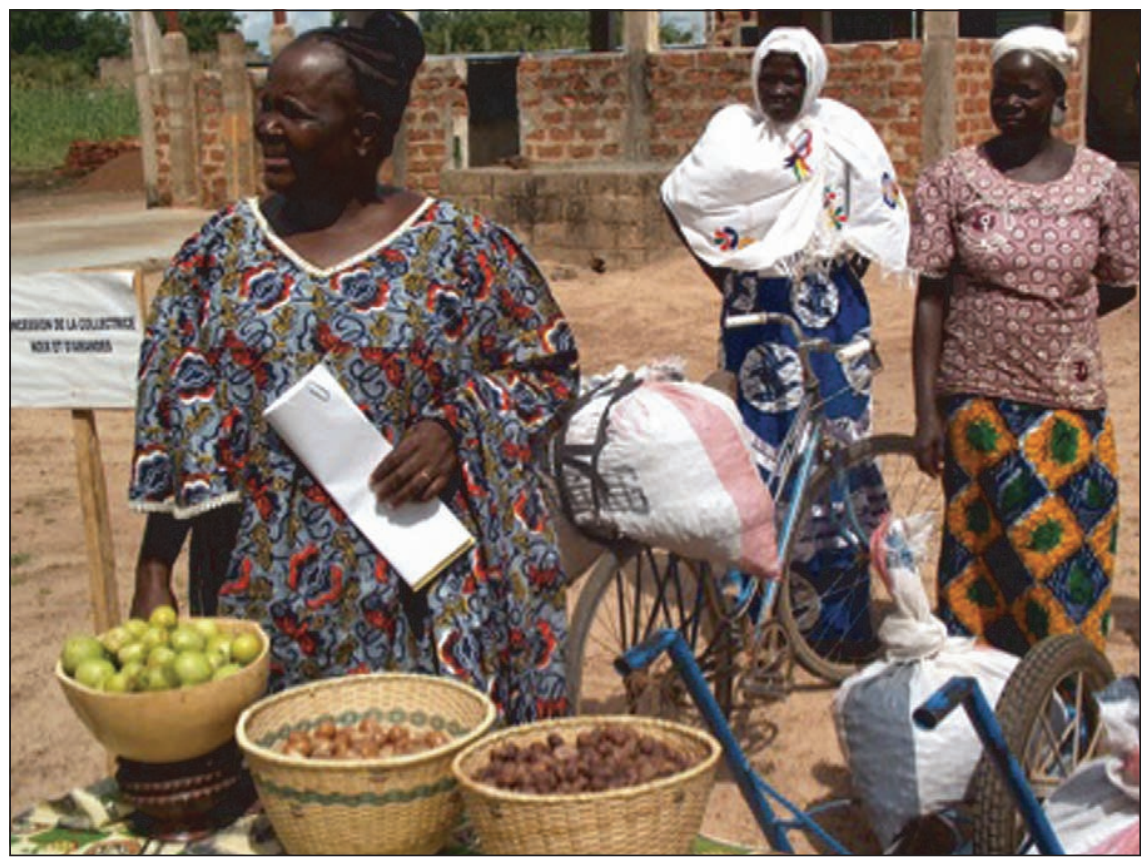

Figure 4. Henriette Ouedraougo présidente de I'association RAGUSSI (" ne dort pas » en Mooré).
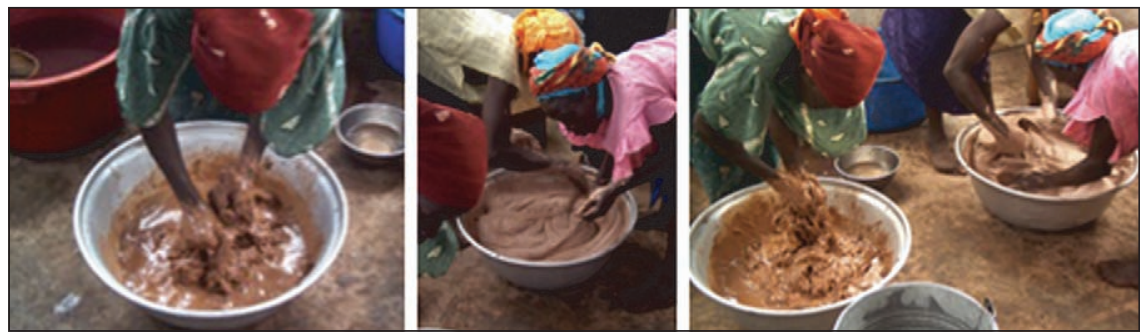

Figure 5. Extraction à l'eau du beurre.
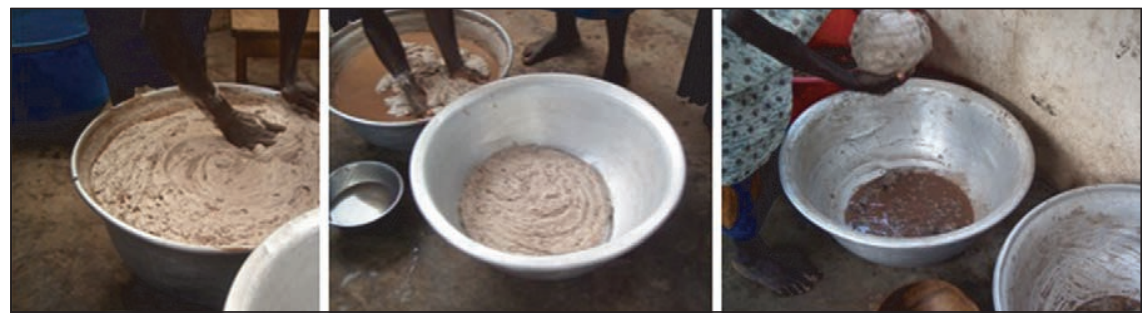

Figure 6. Le lissage du beurre.

Mais ceux-ci n'ont d'intérêt qu'à la condition de respecter le comportement du produit tel que les femmes savent le maîtriser. Ils doivent en fait être adaptés aux groupements et associations de taille réduite et leur mise en place associée à des programmes de formation adaptés.

Leur conception doit ainsi permettre de conserver le caractère de filières faisant intervenir beaucoup de personnel tout en demeurant faciles à gérer.

\section{Production : I'approche semi- industrielle et industrielle}

"Si pressé que je sois, je ne peux pas dire à mon derrière passe devant moi » (Malinké, Sénégal) »
L'approche semi-industrielle et industrielle relève d'une vision rationnalisée de la production. Les organismes de recherches ont très tôt développé des études pour permettre sa mise en place.

Ainsi dans le premier numéro de la revue Oléagineux (septembre 1946), René Dumont, alors maître de conférence à I'Institut national agronomique, souligne le potentiel de production des oléagineux aux Colonies (figure 7).

La création du GIDOTOM (Groupement Interprofessionnel pour le Développement de la Production des Oléagineux dans les Territoires d'Outre Mer) (1947) et celle d'une commission des corps gras du commissariat général au Plan avec une souscommission des produits concrets de la zone tropicale traduisent la volonté de planifier la production de matières grasses au sortir de la guerre :

Mesures concernant les prix et les importations, le transport, le fret; élaboration de plan d'avenir, mise en place de stations agricoles, d'huileries et d'autres projets: c'est dans ce contexte» que plusieurs stations expérimentales pilotées par I'IRHO sont créées en Afrique dont certaines concernent la production de beurre de karité et la rationalisation de sa transformation, de la collecte des noix à l'huile.

\section{Raffinage du beurre de karité}

Après obtention, le beurre brut est généralement conditionné pour être vendu ; un raffinage est en souvent nécessaire pour répondre aux cahiers des charges des utilisateurs. Ainsi, pour la cosmétique, un beurre blanc et peu odorant est demandé ; dans le domaine alimentaire les caractéristiques souhaitées sont classiquement celles d'une graisse raffinée.

Cette opération de raffinage est le plus souvent réalisée dans les pays importateurs; un point essentiel est de maîtriser l'acidité du beurre brut ; si celle-ci est trop importante, le raffinage va entraîner des pertes énormes, voire devenir irréalisable. D'où la nécessité de contrôler cette acidité dès la production et de sensibiliser les coopératives de production à la maitrise de ce paramètre ; cette maîtrise doit s'exercer dès la collecte des noix et leur stockage avant traitement.

Au niveau des opérations de raffinage proprement dites, celles-ci sont classiques : raffinage physique ou chimique sont possibles : les beurres riches en latex - ceux d'extraction au solvant en particulier - peuvent se révéler très laborieux à raffiner. Le conditionnement acide ou une démucilagination acide est alors vivement recommandé. 


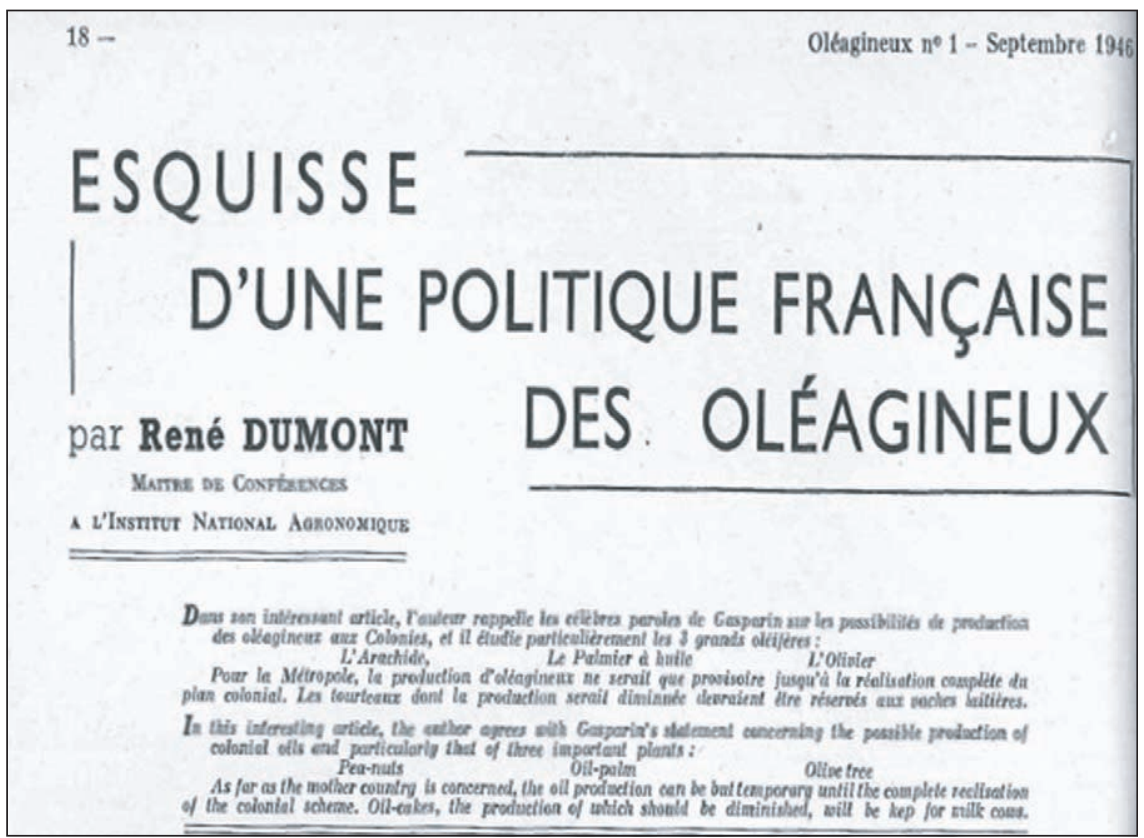

Figure 7. L'article de René Dumont dans le premier numéro de Oléagineux, fait le point sur les potentialités des oléagineux.

\begin{tabular}{|c|c|c|c|}
\hline \multicolumn{4}{|c|}{-Eau, impuretés, acidité : caractéristiques très variables sur beurre brut } \\
\hline - Indice d'iode 58 & \multicolumn{3}{|c|}{ Isapo: $180 \mathrm{mg} / \mathrm{kg}$} \\
\hline \multirow[t]{9}{*}{ - Melting range $35-36{ }^{\circ} \mathrm{C}$} & \multicolumn{3}{|c|}{ insaponifiable de 5 à $15 \%$ (v. mangifolia) } \\
\hline & Acide palmitique & C16:0 & $3,6 \%$ \\
\hline & Acide palmitoléique & C16:1 & $0,1 \%$ \\
\hline & Acide stéarique & C18:0 & $42,5 \%$ \\
\hline & Acide oléique & C181 (n-9) & $45 \%$ \\
\hline & Aide linoleique & $\mathrm{C} 18: 2(\mathrm{n}-6)$ & $6,2 \%$ \\
\hline & Acide linoléique & C18:3 (n-3) & $0,1 \%$ \\
\hline & Acide arachidique & C20:0 & $1,4 \%$ \\
\hline & autres & & $1,1 \%$ \\
\hline
\end{tabular}

Figure 8. Caractéristiques du beurre de karité.

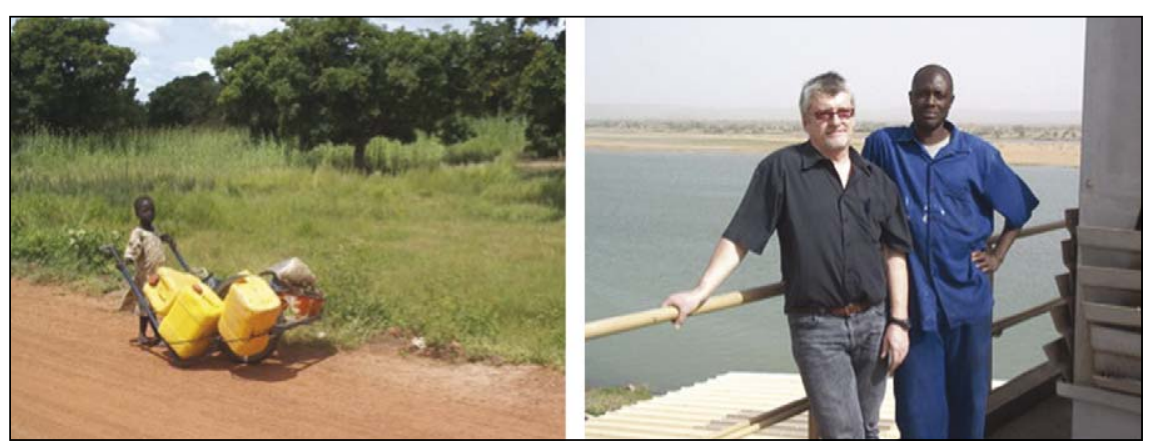

Figure 9. "L'enfant qui n'est jamais sorti de sa maison trouve que c'est sa mère seule qui sait bien assaisonner une sauce » (Minah, Bénin) pour Aisatou!
Si le beurre brut est de bonne qualité (AO $<2 \%$, eau + impuretés $<0,3 \%)$, une simple désodorisation vers $180 / 200{ }^{\circ} \mathrm{C}$ suffit pour obtenir une qualité tout à fait adaptée à la cosmétique ; cette voie doit être privilégiée pour minimiser les coûts de traitement; le beurre brut est avantageusement conditionné dans des fûts à ouverture large pour permettre leur fonte avant raffinage ; le conditionnement en carton avec poche plastique est à ce stade particulièrement mal adapté.

La valorisation suivant une filière bio est aussi un axe intéressant ; il n'y a pas en principe de risque de contamination avec des produits phytosanitaires, les arbres n'étant pas traités ; cependant des contrôles d'absence de pesticides peuvent être recommandés; nous avons déjà pu constater certains cas de contamination de beurre avec de l'endosulfan (coton traité à proximité ?).

Pour illustration, nous donnons ci-après deux exemples de valorisation du beurre de karité.

\section{Exemples de valorisation du beurre de karité}

Le beurre de karité est à la base d'une formule d'un CBE (Cacao Butter Equivalent) de très bonnes caractéristiques comme le démontrent notamment les courbes de Prichard ainsi que les analyses par DSC (Differential Scanning Calorimetry). Le substitut BK 778 mis au point et breveté en 1978 par la société BLOHORN est réalisé ainsi : le mélange de matières grasses de départ se compose de $40 \%$ de beurre de karité raffiné et de $60 \%$ de stéarine de palme de deuxième fractionnement en phase solvant d'une oléine de palme classique (II 58) ou d'une stéarine de palme particulière obtenue par fractionnement en phase huile. Ce mélange est fractionné dans I'hexane et donne $30 \%$ d'une fraction concrète qui constitue le $\mathrm{CBE}$ BK 778.

\section{Enrichissement en insaponifiable de beurre de karité}

Comme évoqué plus haut, I'utilisation du beurre de karité dans l'industrie de la cosmétologie reste une voie intéressante de valorisation.

Le beurre de karité présente une teneur élevée en insaponifiable (de 5 à $8 \%$ en moyenne, jusqu'à $15 \%$ ).

Sa composition est la suivante :

- Alcools triterpéniques (65\%) sous forme d'esters de l'acide cinnamique - cas unique ou d'esters d'acides gras :

- butyrospermol (12/20 \%);

- lupeol (17/28\%);

- alpha (7/10 \%) et bêta (38/55 \%) amyrine ;

- stérols (5\%); Methyl stérols (2\%) 
- hydrocarbures (30 \%) karitène : hydrocarbure insaturé $\mathrm{C} 32 \mathrm{H} 56$ isoprénique existant sous 4 formes isomères.

Le beurre de karité contient peu de tocophérols (90/150 ppm) mais sa composition en acides gras plutôt saturés lui confère néanmoins une bonne résistance à l'oxydation. L'intérêt en cosmétique résidant dans l'insaponifiable, on s'est logiquement intéressé à enrichir en insaponifiable les huiles et beurre qui en contiennent ; diverses méthodes ayant fait l'objet ou non d'industrialisation ont été testées : ainsi la distillation moléculaire qui permet d'obtenir des fractions riches en insaponifiable à partir de soja. L'ITERG s'est intéressé aux techniques d'enrichissement en insaponifiable du beurre de karité sur la demande de la société DESLOG et a développé et breveté en 1992 une technique basée sur la cristallisation en milieu solvant (Prix de l'innovation de Genève en 1992). La matière de départ est du beurre de karité neutralisé, décoloré (insaponifiable 6,4\%) qui est solubilisé dans acétone (dilution $20 \%$ ). La solubilisation se fait à chaud et on récupère une première fraction insoluble à chaud constituée de gommes riches en karitène $2-5 \%$, fraction qui est écartée par soutirage. La partie soluble est refroidie sous agitation à très basse température $\left(-30^{\circ} \mathrm{C}\right)$ et maturée à cette température pendant 12 heures environ.

On procède ensuite à une filtration de la partie oléine et stéarine : environ $10 \%$ d'une fraction liquide à $35 \%$ d'insaponifiable est ainsi récupérée ainsi qu'une fraction concrète et $90 \%$.

Les gommes issues de l'insoluble à chaud sont remélangées dans « l'oléine » et on obtient au final $15 \%$ d'oléine à $48 \%$ d'insaponifiable. Le «Cryokaritène » obtenu a été testé et des propriétés supérieures au beurre de karité ont été démontrées, notamment un pouvoir adoucissant, un pouvoir cicatrisant, une activité anti-érythémateuse et une activité anti-élastase (figure 8).

\section{RÉFÉRENCES}

1. Losch $B$. La libéralisation de la filière cacaoyère ivoirienne et les recompositions du marché mondial du cacao : vers la fin des « pays producteurs » et du marché international ? OCL $2001 ; 8: 562-5$.

2. André E. Le beurre de karité. Oléagineux 1947 ; $2: 546-52$.

3. Labat RP. Nouvelle relation de l'Afrique Occidentale. D'après les mémoire d'André Brûe, vol. III, p. 345.

4. Mungo Park. Voyage dans l'intérieur de l'Afrique. Première édition: 1797. Paris: Ed. François Maspéro, Coll. La découverte, 1980.

\section{POUR EN SAVOIR PLUS}

Cabakulu M. Dictionnaire des proverbes africains. Publication du CERVA, Ed. L'Harmatan, Paris 1992.

De Belsunce. Essais divers sur le beurre de Karité. Bulletin des Matières Grasses 1926: 55-65, 195-198.

Direction des affaires économiques, Secrétariat d'Etat aux colonies. Les exportations agricoles des cercles de l'Afrique occidentale et du Togo français, 1944, Paris.

Dumont R, Paquet C. Démocratie pour I'Afrique. Paris : Editions du Seuil, 1991.

FAO, Organisation des Nations Unies pour I'Alimentation et l'Agriculture, « Amélioration des procédés traditionnels de transformation de certains oléagineux et du manioc», (disponible au GRET).

Graille J, et al. Oléagineux $1988 ; 43: 188$.

GRET (Groupe de Recherche et d'Echange Technologique, association professionnelle de solidarité et de coopération internationale) : fonds documentaire important sur les productions agricoles mondiales, les technologie de transformations, etc., Campus Jardin Tropical, 45 bis Av de la belle Gabrielle, Nogent sur Marne (www.gret. org). En prime, une promenade insolite à Paris, le GRET étant situé dans le bois de Vincennes au milieu des anciens pavillons, vestiges de l'exposition coloniale de 1901.
Hampate BA Amadou. Sur les traces d'Amkoullel I'enfant Peul. Actes Sud, 1998.

Hampate BA Amadou. L'étrange destin de Wangrin. Paris: Presses de la Cité, Collection 10-18, 1973.

Hampate BA Amadou. Vie et enseignement de TiernoBokar, le sage de Bandiagara. Collection «Points Sagesses. Paris: Editions du Seuil, 1980.

Kapseu C, et al. Application du procédé séchagefriture aux amandes de karité : cinétique de séchage. OCL $2004 ; 11$.

Ladurelle H. Le beurre de karité. Thèse, 1984 (cote Iterg CA1 160).

Laur J, Rossignol Castera A, Pages X. Procédé de préparation de fractions de matières grasses d'origine végétale enrichies en matières insaponifiables. Brevet DESLOG (BF 9303226).

L'Illustration, L'Album de l'exposition coloniale, juillet 1931.

Lovett Peter N. Shea Butter Industry expanding in West Africa. Inform 2005 : 16.

Orsenna E. Voyage aux pays du coton. Paris : Editions Fayard, 2006.

Pages X, Musso S, Lehen-Ferrenbach C, et al. Cacao butter substitute and its use for chocolate. Société BLOHORN (Brevets FR-2464655, GB2084170 A, NL-8005299 A).

Pontillon J. Cacao et chocolat, production, utilisation, caractéristiques. Lavoisier, Tecres et à la carte... Edition Le Cèdre, B7622, Laplaigne.

Tano-Debrah Kwaku, Yoshiyuki Ohta. EnzymeAssisted Aqueous Extraction of Shea Fat: a Rural Approach. JAOCS 1995, vol 72, n².

Tano-Debrah Kwaku, Yoshiyuki Ohta. EnzymeAssisted Aqueous Extraction of Fat from Kernels of the Shea Tree, ButyrospermumParkii. JAOCS $1994 ; 71, \mathrm{n}^{\circ} 9$.

Wong Soon, 1988, "The chocolaty fat from the borneoillipe trees".

X., "Arbres africains et cosmétiques», Bulletin TPO (Réseau technologique et partenariat en agroalimentaire), 1994, vol 9 (sept).

Exposition « Femmes dans les arts d'Afrique », musée DAPPER, rue Paul Valery 75016 Paris du 10/10/2008 au 12/07/2009. 